\title{
ESTUDIO PRELIMINAR DE LOS DÍPTEROS ASOCIADOS A CEBOS DE ESTIÉRCOL Y CARROÑA EN UN BOSQUE SERRANO DE SIERRA DE MINAS, URUGUAY
}

\author{
Mónica REMEDiOS, MARÍa MARTÍNEZ \& PATRICIA GONZÁLEZ-VAINER \\ Sección Entomología. Facultad de Ciencias. Iguá 4225. CP11400 Montevideo, Uruguay. \\ $<$ monicaremedios@gmail.com>
}

\begin{abstract}
Remedios, M., M. Martínez \& P. González-Vainer. 2012. Estudio preliminar de los dípteros asociados a cebos de estiércol y carroña en un bosque serrano de Sierra de Minas, Uruguay. Acta Zoológica Mexicana (n. s.), 28(2): 378-390.

RESUMEN. Se realizó un estudio preliminar sobre la estructura de los ensambles de dípteros coprófilos y necrófilos a nivel de familias, en un bosque serrano en Sierra de Minas, Uruguay. Asimismo se estableció la variación estacional de dicha estructura para ambos grupos de dípteros. Se realizaron muestreos mensuales durante un año, colocando durante una semana, seis trampas de caída, tres cebadas con estiércol vacuno y tres con hígado vacuno. Se recolectaron 3,142 individuos pertenecientes a 21 familias del Orden Diptera. Se registró por primera vez la familia Heleomyizidae en Uruguay. Las familias con mayor abundancia fueron Phoridae y Sphaeroceridae, representando el 58\% del total de ejemplares recolectados. En las trampas con estiércol vacuno predominaron Sphaeroceridae (42\%), Cecidomyiidae (14\%) y Phoridae (12\%). En las necrotrampas la familia más abundante fue Phoridae que representó el $59 \%$ del total de individuos, seguida de Drosophilidae (10\%). Los dos ensambles presentaron el mismo patrón de variación estacional de la abundancia, con picos en otoño (marzo-abril) y en primavera (octubre-noviembre). La riqueza de familias del ensamble coprófilo fue mayor durante la primavera (octubre a diciembre) y en otoño (abril) mientras que el ensamble necrófilo presentó la mayor riqueza de familias solamente de octubre a noviembre. Este estudio constituye un primer paso en la generación de conocimientos acerca de la composición de las comunidades de dípteros en ambientes nativos en Uruguay.

Palabras clave: coprotrampa, necrotrampa, Phoridae, Sphaeroceridae, Heleomyzidae, comunidad.
\end{abstract}

Remedios, M., M. Martínez \& P. González-Vainer. 2012. A preliminar study on dipteran associated with dung and carrion baits in a serrania forest in Sierra de Minas, Uruguay. Acta Zoológica Mexicana (n. s.), 28(2): 378-390.

ABSTRACT. A preliminary study on coprophilous and necrophilous Diptera assemblages was done at family level, in a serranía forest in Sierra de Minas, Uruguay. Besides, seasonal structure variations of both assemblages were assessed. Dipterous were sampled monthly during a year, using pitfall traps baited with cow dung and cow liver, which were deployed for a week. A total of 3,142 individuals corresponding to 21 families, were captured. Heleomyzidae was recorded for the first time in Uruguay. The most abundant families were Phoridae and Sphaeroceridae which represented $58 \%$ of total number

Recibido: 25/10/2011; aceptado: 27/04/2012. 
of captures. Sphaeroceridae (42\%) was the dominant family in dung baited traps, followed by Cecidomyiidae (14\%) and Phoridae (12\%). Phoridae (59\%) was the most abundant family in carrion baited traps, followed by Drosophilidae (10\%). Both assemblages showed the same pattern of seasonal abundances variation, and revealed peaks in spring (October - November) and in autumn (April). The number of families of coprophilous flies was greater during spring (October-December) and in autumn (April) while necrophilous flies showed the highest richness of families only from October to November. This study represents the contribution to the knowledge about dipteran communities in native ecosystems in Uruguay.

Key words: dung baited pitfall trap, carrion baited pitfall trap, Phoridae, Sphaeroceridae, Heleomyzidae, community.

\section{INTRODUCCIÓN}

Diptera es uno de los órdenes de insectos de mayor riqueza; comprende más de 120.000 especies conocidas y es el cuarto más numeroso después de Coleoptera, Lepidoptera e Hymenoptera (Bentancourt et al. 2009). Estos insectos constituyen un grupo con una alta variedad morfológica y ecológica desempeñando un rol fundamental en todos los ecosistemas. Poseen hábitos alimentarios muy diversos, existiendo especies fitófagas, detritívoras de materia orgánica de origen vegetal y animal, polinizadoras, predadoras, parasitoides y hematófagas (Hernández-Ortiz \& Dzul-Cauich 2008). Varias especies pueden ser vectores de diversas enfermedades humanas y animales y algunas pueden ser productoras de miasis tanto en el hombre como en animales domésticos (Greenberg 1971, Greenberg 1973, Guimarães et al. 1983); también hay numerosos grupos de dípteros que provocan daños en cultivos y productos almacenados (Carles-Tolrá 1997). Por estos motivos, los estudios ecológicos sobre dípteros se han enfocado, tradicionalmente, en grupos de especies relacionados con establecimientos urbanos y agrícola-ganaderos (Carles-Tolrá 1997, Mulieri et al. 2006). Sin embargo, a partir del nuevo siglo, se han incrementado los estudios ecológicos centrados en la utilidad de los dípteros como indicadores de diversidad y del grado de degradación de los ambientes naturales por las actividades antropogénicas (Kearns 2001, Centeno et al. 2004, Dziock 2006, Pollet 2009, Bizzo et al. 2010).

Los dípteros constituyen, junto a los coleópteros, uno de los grupos predominantes dentro de las comunidades de insectos del estiércol y la carroña, y cumplen un rol fundamental en el proceso de descomposición de estos productos de origen animal (Putman 1983). Los dípteros explotan rápidamente estos recursos, encontrándose especies coprófagas, necrófagas, predadoras y también omnívoras (Putman 1983, Hanski 1987, Cabrera Walsh \& Cordo 1997). Los excrementos y cadáveres son utilizados por las especies coprófagas y necrófagas como fuente de proteínas para la maduración de sus huevos, como sustrato para oviponer y/o como alimento para el desarrollo de las larvas (Kuusela \& Hanski 1982; Martínez Sánchez et al. 2000a). La acción de los dípteros detritívoros es de vital importancia en el reciclado de los nutrientes y la energía contenidos en estos recursos, pues prepara el sustrato para la intervención de 
los verdaderos descomponedores finales que son los hongos y bacterias (Galante \& Marcos-García 1997).

En Uruguay, los estudios realizados en dípteros se han centrado principalmente en aquellos grupos de especies de interés sanitario, ya sea médico o veterinario (Carballo et al. 1990, Spinelli \& Martínez 1991, Salvatella 1997, Martínez 2002, Rossi \& Martínez 2003), y en comunidades de Drosophilidae (Goñi et al. 1997, 1998). No se han realizado hasta la fecha estudios de la biodiversidad o de la composición de las comunidades de dípteros coprófilos y necrófilos en los diferentes ecosistemas presentes en Uruguay.

Varios estudios han examinado la correlación positiva entre la riqueza de taxones superiores y la riqueza total de especies de diversos grupos en diferentes áreas geográficas (Gaston \& Williams 1993; Prance 1994; Williams \& Gaston 1994, Andersen 1997). De acuerdo con Andersen (1997), el poder de predicción de la riqueza de géneros o de taxones superiores disminuye a medida que aumenta la escala espacial pero de igual manera la relación tiende a ser más fuerte en sitios que representan un mismo tipo de hábitat. En conclusión, el uso de sustitutos para la riqueza total de especies es posible pero limitado a una escala local y dentro de un mismo tipo de hábitat. La determinación de la composición de la fauna a nivel de taxones de alto rango tiene una ventaja por su bajo costo y su fácil realización, en comparación con la determinación de la riqueza de especies (Williams \& Gaston 1994). En este sentido, de acuerdo con Hernández-Ortiz \& Dzul-Cauich (2008), el uso de los Diptera basado en la identificación de familias tiene un alto potencial para evaluar la biodiversidad de ciertos ecosistemas.

El objetivo de este trabajo es determinar la composición, a nivel de familias, de los ensambles de dípteros coprófilos y necrófilos y su variación estacional en un ecosistema nativo de Uruguay, el bosque serrano.

\section{MATERIALES Y MÉTODOS}

Área de estudio. El bosque de sierras en Uruguay representa en cuanto a extensión, la segunda formación boscosa más importante luego del bosque ribereño; se desarrolla en áreas pedregosas que incluyen cerros, sierras y asperezas, normalmente asociados a cursos de agua y manantiales (Carrere 2001). Históricamente el bosque nativo se ha utilizado como fuente de madera para combustible o construcciones rústicas y ha estado sometido a una fuerte presión de tala que hoy se traduce en la existencia de bosques primarios fragmentados y reducidos en su extensión (80.000 ha) (Porcile Maderi 2005). A pesar de su fragmentación, estos bosques son ecosistemas con una alta biodiversidad, existiendo un profundo conocimiento de la flora y fauna, particularmente de reptiles, aves y mamíferos (Evia \& Gudynas 2000), no así en relación a la fauna de artrópodos (Carrere 2001). El sitio particular de muestreo se ubicó en la ladera oriental del Cerro de la Virgen, Sierra de Minas, Departamento de Lavalleja, en el Km 
102 de la Ruta 81, (34³0’59’'S, 55²0’07’’W). Este cerro está cubierto por una gran extensión de bosque serrano de vegetación densa, con arbustos y árboles de hasta $3 \mathrm{~m}$ de altura de diversas especies, con helechos y epífitas asociados. Los principales representantes de la flora son especies espinosas como: Scutia buxifilia, Celtis spinosa, Schinus longuifolius, Colletia paradoxa, Zanthoxylum rhoifolium, Citharexylum montevidense, Lithrae brasiliensis y Lithrae molleidoes y algunas plantas inermes como Myrsine laetevirens, Myrsine coriacea, Pouteria salicifola, Blepharocalyx salicifolius, Daphnopsis racemosa y Dadonaea viscosa (Costa \& Miranda 2007).

Muestreo. El muestreo se realizó mensualmente durante 13 meses (mayo/2002mayo/2003). Se colocaron seis trampas de caída, tres cebadas con estiércol vacuno fresco (coprotrampas) y tres con hígado vacuno (necrotrampas), completando un total de 78 trampas, considerando cada trampa una muestra. Las coprotrampas consistían en un recipiente plástico de $12 \mathrm{~cm}$ de diámetro $\times 10 \mathrm{~cm}$ de altura, el cual se enterraba a ras de suelo, colocando encima una malla metálica de $5 \mathrm{~cm}$ de apertura, que sostenía 450 gr de estiércol vacuno envuelto en gasa. Las necrotrampas tenían un diseño diferente para evitar el robo del cebo por parte de animales carroñeros. Estaban compuestas por dos recipientes, cada uno de $10 \mathrm{~cm}$ de diámetro $\times 9 \mathrm{~cm}$ de altura, unidos uno sobre el otro y comunicados entre sí por orificios. En el recipiente inferior se colocaba el liquido conservante y en el superior el cebo de carroña (20 gr). Este último tenía una tapa perforada que permitía la entrada de los insectos y en el fondo tenía orificios que permitían la caída de los mismos en el líquido conservante. Fueron enterradas a ras de suelo y protegidas con una malla metálica. Se utilizó formol al 5\% con unas gotas de detergente en ambos tipos de trampas como liquido conservante. Las trampas se colocaron durante una semana, disponiéndose en forma alternada a lo largo de una transecta separadas 20 m entre sí.

Los datos meteorológicos (temperatura media y precipitaciones) fueron registrados por la Estación Meteorológica de Solís situada a 20 km de la zona de estudio y fueron cedidos por la Dirección Nacional de Meteorología.

Procesamiento de muestras y análisis de datos. Para la identificación de los dípteros a nivel de familia se utilizaron las claves de Carles-Tolrá (2004), Borror et al. (1992) y McAlpine et al. (1981). Se determinaron las abundancias absolutas y relativas, anuales y mensuales, de las familias de dípteros capturadas con los dos tipos de trampas para establecer la estructura de los ensambles y la variación estacional de las mismas. Se aplicó un análisis de similitud (ANOSIM) de 1 vía para determinar diferencias en la composición entre ambos ensambles, construyendo previamente una matriz de similitud entre muestras, utilizando el coeficiente de Bray-Curtis, previa transformación de los datos en raíz cuarta. La diversidad alfa de cada ensamble fue analizada a través de la riqueza de familias. Se determinaron las correlaciones entre las abundancias y riquezas mensuales de los dípteros capturados en ambos tipos de trampas y las temperaturas medias y precipitaciones mensuales. 


\section{RESULTADOS}

Se recolectaron 3,142 imagos pertenecientes a 21 familias del Orden Diptera, 1,993 ejemplares (63.4\%) en las coprotrampas y 1,149 individuos (36.6\%) en las necrotrampas. Las familias predominantes fueron Phoridae y Sphaeroceridae que representaron cada una el $29 \%$ del total de ejemplares recolectados. En orden de importancia siguieron Cecidomyiidae con un 8\% y Drosophilidae con un 6\% (Cuadro 1). La composición de los ensambles de dípteros recolectados con los dos tipos de trampas fue significativamente diferente (ANOSIM, $\mathrm{R}=0.40 ; \mathrm{p}=0.001$ ). Sólo nueve familias del

Cuadro 1.Abundancias absolutas (AA) y relativas (AR) de los dípteros recolectados en trampas cebadas con estiércol (CT) y con carroña (NT) en un bosque serrano en Sierra de Minas, Uruguay

\begin{tabular}{|c|c|c|c|c|c|c|}
\hline \multirow[t]{2}{*}{ Familias } & \multicolumn{2}{|c|}{$\mathrm{CT}$} & \multicolumn{2}{|c|}{ NT } & \multicolumn{2}{|c|}{ Totales } \\
\hline & AA & AR (\%) & $\mathrm{AA}$ & AR (\%) & AA & AR (\%) \\
\hline \multicolumn{7}{|c|}{ Suborden Nematocera } \\
\hline Tipulidae & 15 & 0.80 & 0 & 0 & 15 & 0.5 \\
\hline Mycetophilidae & 105 & 5.30 & 3 & 0.3 & 108 & 3.44 \\
\hline Sciaridae & 141 & 7.10 & 5 & 0.4 & 146 & 4.65 \\
\hline Cecidomyiidae & 276 & 13.80 & 0 & 0 & 276 & 8.78 \\
\hline Psychodidae & 28 & 1.40 & 0 & 0 & 28 & 0.89 \\
\hline Ceratopogonidae & 1 & 0.10 & 0 & 0 & 1 & 0.03 \\
\hline Chironomidae & 1 & 0.10 & 0 & 0 & 1 & 0.03 \\
\hline \multicolumn{7}{|c|}{ Suborden Brachycera } \\
\hline Empididae & 2 & 0.10 & 0 & 0 & 2 & 0.06 \\
\hline Dolichopodidae & 2 & 0.10 & 0 & 0 & 2 & 0.06 \\
\hline Phoridae & 229 & 11.50 & 681 & 59.3 & 910 & 28.96 \\
\hline Piophilidae & 3 & 0.20 & 0 & 0 & 3 & 0.09 \\
\hline Anthomyzidae & 1 & 0.10 & 0 & 0 & 1 & 0.03 \\
\hline Sepsidae & 1 & 0.10 & 0 & 0 & 1 & 0.03 \\
\hline Heleomyzidae & 96 & 4.80 & 92 & 8.0 & 188 & 5.98 \\
\hline Sphaeroceridae & 840 & 42.00 & 83 & 7.2 & 923 & 29.34 \\
\hline Drosophilidae & 97 & 4.9 & 113 & 9.8 & 210 & 6.68 \\
\hline Muscidae & 142 & 7.1 & 43 & 3.7 & 185 & 5.89 \\
\hline Fannidae & 0 & 0 & 12 & 1.0 & 12 & 0.38 \\
\hline Calliphoridae & 5 & 0.3 & 84 & 7.3 & 89 & 2.83 \\
\hline Sarcophagidae & 8 & 0.4 & 32 & 2.8 & 40 & 1.27 \\
\hline Tachinidae & 0 & 0 & 1 & 0.1 & 1 & 0.03 \\
\hline Total & 1993 & 100 & 1149 & 100 & 3142 & 100 \\
\hline
\end{tabular}


total se recolectaron en los dos tipos de trampas, diez se encontraron solamente en las coprotrampas y dos, Fannidae y Tachinidae, fueron recolectadas exclusivamente en las necrotrampas. Las familias más abundantes capturadas en las coprotrampas fueron Sphaeroceridae, Cecidomyiidae, Phoridae, Muscidae y Sciaridae. Por otra parte, en las necrotrampas predominaron Phoridae y Drosophilidae (Cuadro 1). El ensamble de dípteros recolectado con las coprotrampas fue más abundante y con mayor riqueza de familias (19) que el ensamble recogido en las necrotrampas (11 familias).

El ensamble de dípteros capturado en las coprotrampas mostró dos picos de abundancia: uno en primavera (octubre-noviembre) y otro en otoño (abril) siendo baja la abundancia en los meses de verano y casi nula en los meses de invierno (Fig. 1). En el pico de primavera predominó Sphaeroceridae mientras que Muscidae y Phoridae predominaron en el pico de otoño (Fig. 2).

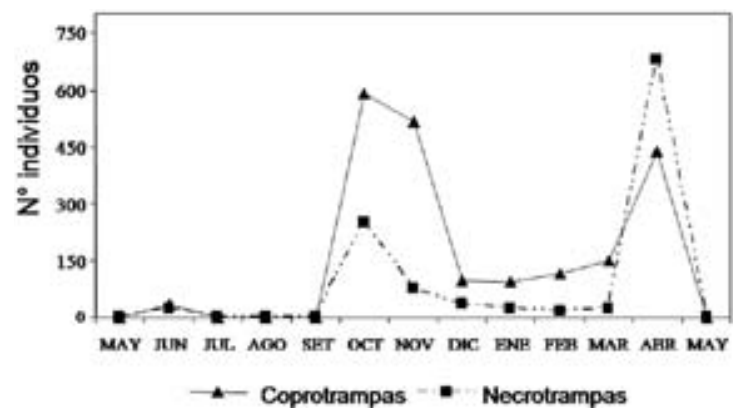

Figura 1. Variación estacional de la abundancia de los dípteros coprófilos y necrófilos capturados en un bosque serrano en Sierra de Minas, Uruguay desde Mayo/2002 a Mayo/2003.

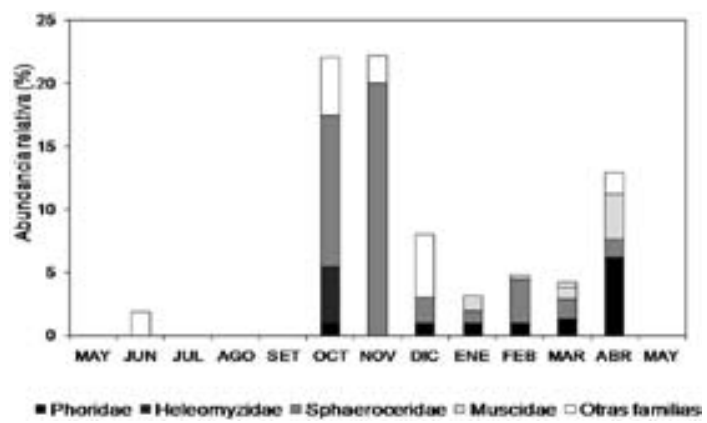

Figura 2. Variación estacional de la abundancia relativa de las familias de dípteros capturadas en trampas cebadas con estiércol en un bosque serrano en Sierra de Minas, desde Mayo/2002 a Mayo/2003. 
El ensamble de dípteros capturados en las necrotrampas presentó dos picos de abundancia, uno en primavera (octubre) y otro en otoño (abril), siendo éste marcadamente superior al primero (Fig.1). La abundancia fue baja el resto de los meses de primavera y verano y casi nula en invierno. En el pico de octubre predominaron Phoridae, Heleomyzidae y Sphaeroceridae en proporciones similares, mientras que en el pico de abril Phoridae fue la familia dominante (Fig. 3).

La riqueza de familias en las coprotrampas fue mayor en primavera (octubre a diciembre) y en otoño (abril) mientras que, en las necrotrampas, se recolectó la mayor riqueza de familias sólo en primavera (octubre y noviembre (Fig. 4).

La precipitación anual total de Mayo de 2002 a Mayo de 2003 fue de $1573 \mathrm{~mm}$. La temperatura media más alta ocurrió en Enero de $2003\left(23.2^{\circ} \mathrm{C}\right)$ y el valor más bajo en Julio de $2002\left(10.6^{\circ} \mathrm{C}\right)$ (Fig. 5). La abundancia y la riqueza de familias de dípteros coprófilos estuvieron positivamente correlacionadas con las temperaturas medias

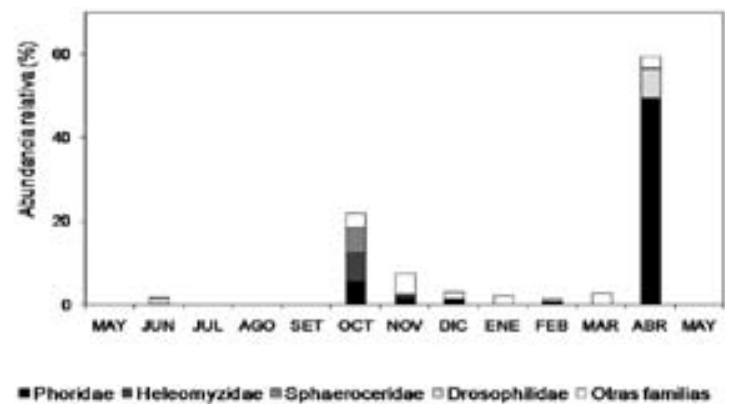

Figura 3. Variación estacional de la abundancia relativa de las familias de dípteros capturadas en trampas cebadas con carroña en un bosque serrano en Sierra de Minas, desde Mayo/2002 a Mayo/2003.

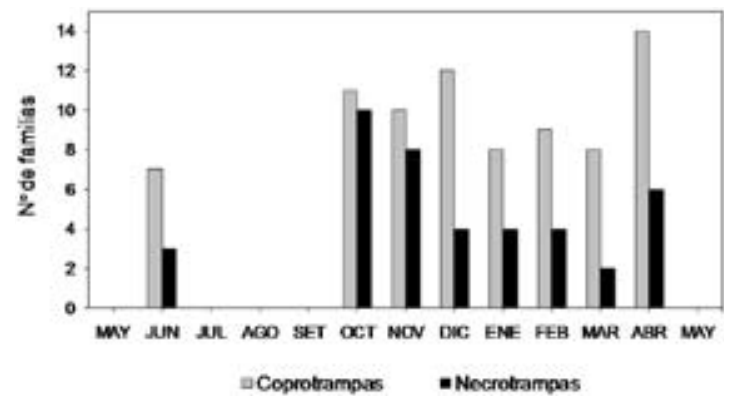

Figura 4. Variación estacional de la riqueza de familias capturada en trampas cebadas con estiércol y carroña en un bosque serrano en Sierra de Minas, desde Mayo/2002 a Mayo/2003. 


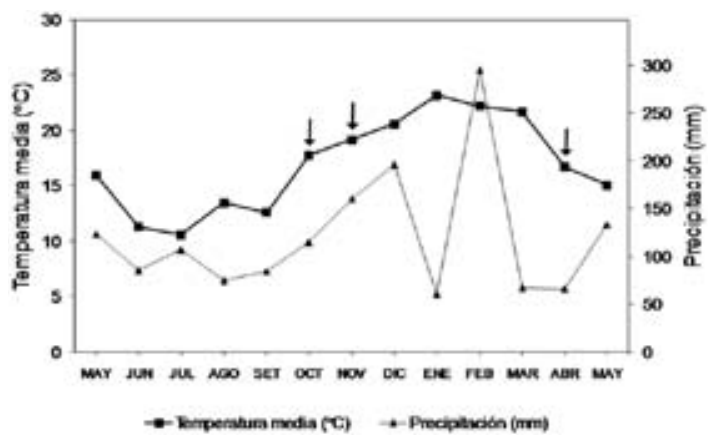

Figura 5. Datos meteorológicos tomados por la Estación Meteorológica Solís, Departamento de Lavalleja, Uruguay, desde Mayo/2002 a Mayo/2003. Las flechas indican los períodos en que se producen los picos de abundancia de dípteros en las coprotrampas y necrotrampas en un bosque serrano en Sierra de Minas.

mensuales, no así con las precipitaciones. En el caso de los dípteros necrófilos, sólo la riqueza de familias estuvo positivamente correlacionada con la temperatura media mensual (Cuadro 2). Ambos ensambles presentaron valores máximos de abundancia y riqueza de familias a temperaturas medias que oscilaron entre 17 y $21^{\circ} \mathrm{C}$.

\section{DISCUSIÓN}

El número y la composición de familias recolectadas con las necrotrampas fueron consistentes con los encontrados en estudios realizados en México con trampas NTP80 (necrotrampa permanente, modelo 1980) enterradas en el suelo (Morón \& Terrón 1984, Deloya et al. 1987). La gran proporción de Phoridae en las necrotrampas también es un patrón recurrente en los estudios mencionados y en el realizado por Hernández-Ortiz \& Dzul-Cauich (2008). Las larvas de esta familia se desarrollan

Cuadro 2. Resultados de las correlaciones de Spearman por rangos, entre abundancia y riqueza de familias de dípteros y algunas variables ambientales

\begin{tabular}{lcccc}
\hline & \multicolumn{2}{c}{ Temperatura media } & \multicolumn{2}{c}{ Precipitación } \\
\hline Coprotrampas & $\mathrm{R}$ & $\mathrm{p}$ & $\mathrm{R}$ & $\mathrm{p}$ \\
$\quad$ Abundancia & & & & \\
$\quad$ Número de familias & 0.62 & $0.02^{*}$ & 0.07 & 0.83 \\
Necrotrampas & 0.61 & $0.02^{*}$ & 0.11 & 0.73 \\
$\quad$ Abundancia & & & & \\
$\quad$ Número de familias & 0.51 & 0.08 & -0.07 & 0.83 \\
\hline
\end{tabular}


típicamente en materia orgánica en descomposición, ya sea de origen animal o vegetal y algunas especies están estrechamente asociadas a cadáveres, incluso enterrados (Byrd \& Castner 2001, Battán Horestein et al. 2010).

Todas las familias recolectadas en las coprotrampas con abundancias próximas a 100 individuos o más, excepto Drosophilidae, tienen especies con hábitos coprófilos conocidos, presentando larvas coprófagas, como es el caso de Sphaeroceridae, Mycetophilidae, Siaridae, Cecidomyiidae, Psychodidae y Muscidae o larvas predadoras de otras especies coprófagas como es el caso de algunos Muscidae, por lo cual las hembras acuden a los excrementos a oviponer (Hanski 1987, Cabrera Walsh \& Cordo 1997, Bentancourt et al. 2009).

Heleomyzidae, cuya abundancia fue similar en ambos tipos de trampas, posee algunas especies que en estado adulto son atraídas tanto por el estiércol como por la carroña, medios en los cuales oviponen y en los que se desarrollarán sus larvas posteriormente (McAlpine 2007). Esta familia se registra por primera vez en Uruguay.

La presencia casi equitativa de Drosophilidae en ambos tipos de trampas podría deberse, considerando la biología de sus especies, a la atracción ejercida por los gases desprendidos por el líquido conservante (formol al 5\%) más que a la atracción por el estiércol o la carroña (Morón \& Terrón 1984). Sin embargo, no se debe descartar que alguna especie haya sido atraída por el cebo de carroña, ya que algunas especies de Drosophila se han asociado a cuerpos de cerdo en descomposición (Moura et al. 1997, Carvalho et al. 2000)

Los dípteros Calliphoridae y Sarcophagidae, típicamente coprófagos y necrófagos, presentaron abundancias muy bajas si se compara con los resultados de otros estudios en la región que han utilizado como cebo hígado vacuno y heces de perro, pero aplicando como método de recolección la red entomológica sobre el cebo (Mulieri et al. 2006, Mariluis et al. 2007). Es probable que el método de captura empleado en este estudio, la trampa de caída, no sea apropiado para estos dípteros, ya que permite fácilmente el escape de los imagos. El estudio realizado por Morón \& Terrón (1984) con necrotrampas enterradas en el suelo también reveló bajas abundancias relativas de estas familias. Por otra parte, en el caso de la coprotrampa, el bajo número de individuos pudo deberse también al tipo de cebo utilizado, ya que la importancia relativa de califóridos y sarcofágidos en las heces de herbívoros es escasa (Skidmore 1991), ocurriendo lo contrario en las heces de vertebrados carnívoros y omnívoros (Martínez-Sánchez et al. 2000b). En el caso de la necrotrampa, el tamaño y el tipo de cebo también pudieron haber influido negativamente en la abundancia de dípteros necrófagos recolectados. Algunos estudios sobre coleópteros necrófagos han demostrado que trozos de animales en descomposición (e.g. hígado vacuno, vísceras de pollo o trozos de pescado) son menos atractivos para estos insectos, que las carcasas de cuerpos enteros (Rafcliffe 1996, Rintoul et al. 2005). El mismo efecto podría ocurrir para los dípteros. De hecho, algunas especies de dípteros califóridos acuden a oviponer en 
mayor abundancia a grandes cadáveres que a carcasas de pequeños animales (BattánHorenstein et al. 2005, Battán-Horenstein et al. 2007).

Ambos ensambles presentaron una marcada estacionalidad, siendo más abundantes en primavera (octubre y noviembre) y en otoño (abril), meses caracterizados por temperaturas templadas ( 17 a $21^{\circ} \mathrm{C}$ ); esto sugiere que los adultos son sensibles y menos tolerantes a las temperaturas altas y bajas del verano e invierno respectivamente. Este patrón de variación estacional de la abundancia también se ha observado en otros insectos en Uruguay, tal es el caso de los ensambles de coleópteros coprófagos (Morelli et al. 2002) y es consistente con el hallado en la Provincia de Buenos Aires para comunidades de Calliphoridae (Mulieri et al. 2006).

Dada la importancia de los dípteros como descomponedores de materia orgánica, como potenciales indicadores de biodiversidad y de influencia antrópica, es necesario profundizar en el estudio de estos ensambles a nivel de especies, para tener un conocimiento cualitativo y cuantitativo de la riqueza de dípteros del bosque autóctono. Por otra parte, para tener un conocimiento más completo de las comunidades de dípteros coprófilos y necrófilos será necesario emplear métodos de captura más adecuados, como la aplicación de la red entomológica directamente sobre el cebo, trampas WOT (Vogt et al. 1985, Martínez-Sánchez et al. 2000b) y diferentes tipos de cebo, incluyendo excrementos de mamíferos carnívoros y omnívoros y cuerpos enteros de animales en descomposición.

Agradecimientos. Agradecemos el apoyo financiero brindado por el Programa de Desarrollo de Ciencias Básicas (PEDECIBA) que permitió realizar el trabajo de campo. También agradecemos los comentarios y sugerencias de los revisores que enriquecieron el manuscrito.

\section{LITERATURA CITADA}

Andersen, A. N. 1997. Using Ants as bioindicators: Multiscale Issues in Ant Community Ecology. Conservation Ecology [online] 1(1): 8. Disponible en Internet. URL: http://www.consecol.org/vol1/ iss1/art8

Battan Horenstein, M., M. I. Arnaldós, B. Rosso \& M. D. García. 2005. Estudio preliminar de la comunidad sarcosaprófaga en Cordoba (Argentina):aplicacion a la entomologia forense. Anales de Biologia, 27: 191-201.

Battan Horestein, M., A. X. Linhares, B. Rosso \& M. D. García. 2007. Species composition and seasonal succession of saprophagous calliphorids in a rural area of Cordoba, Argentina. Biological Research, 40: 163-171.

Battán Horenstein, M., A. X. Linhares, B. Rosso \& M. D. García. 2010. Decomposition and dipteran succession in pig carrion in central Argentina: ecological aspects and their importance in forensic science. Medical and Veterinary Entomology, 24: 16-25.

Bentancourt, C., I. Scatoni \& E. Morelli. 2009. Insectos del Uruguay. Facultad de Agronomía, Facultad de Ciencias, Universidad de la República, Montevideo, 657pp.

Bizzo, L., M. S. Gottschalk, D. C. De Toni \& P. R. P. Hofmann. 2010. Seasonal dynamics of a drosophilid (Diptera) assemblage and its potencial as bioindicator in open environments. Iheringia, 100: 185-191. 
Remedios et al. Dípteros asociados a cebos de estiércol y carroña en Sierra de Minas, Uruguay

Borror, D. J., C. A. Triplehorn \& N. F. Johnson. 1992. An introduction to the study of insects. Harcourt College Publishers, Fort Worth, TX., 875pp.

Byrd J. H. \& J. L. Castner. 2001. Insects of Forensic Importance, pp. 43-75. In: J. H. Byrd \& J. L. Castner (Eds.). Forensic Entomology: The utility of arthropods in legal investigations. CRC Press, Boca Raton. Florida.

Cabrera Walsh, G. \& H. A. Cordo. 1997. Coprophilous arthropod community from Argentina with species of potential use as biocontrol agents against pest flies. Environmental Entomology, 26: 191200.

Carballo, M., A. Colombo \& T. Heinzen. 1990. Presencia de especies de dípteros Califóridos causantes de miasis cutáneas en Uruguay. Relevamiento de larvas parasitarias (instar III) en rumiantes. Veterinaria, 26: 4-6.

Carles-Tolrá, M. 1997. Los dípteros y el hombre. Boletín de la Sociedad Española Aragonesa, 20: 405-425.

Carles-Tolrá, M. 2004. Dípteros, pp. 657-682. In: J. A. Barrientos (Ed.). Curso práctico de Entomología. Asociación Española de Entomología, Barcelona.

Carrere, R. 2001. Monte Indígena. Brecha, Montevideo.101 pp.

Carvalho, L. M., P. J. Thyssen, A. X. Linhares \& F. A. Palhares. 2000. A checklist of arthropods associated with pig carrion and human corpses in Southeastern Brazil. Memórias de Fundação Oswaldo Cruz, 95: 135-138.

Centeno, N., D. Almorza \& C. Arnillas. 2004. Diversity of Calliphoridae (Insecta: Diptera) in Hudson, Argentina. Neotropical Entomology, 33: 387-390.

Costa, B. \& C. Miranda. 2007. Bosques Nativos en el Uruguay, una guía de apoyo para educadores. O. S. Media, Montevideo. 86 pp.

Deloya, C., G. Ruiz-Lizarraga \& M. A. Morón. 1987. Análisis de la entomofauna necrófila en la región de Jojutla, Morelos, México. Folia Entomológica Mexicana, 73: 157-171.

Dziock, F. 2006. Life-history data in bioindication procedures, using the example of hoverflies (Diptera, Syrphidae) in the Elbe Floodplain. International Review of Hydrobiology, 91: 341-363

Evia, G. \& E. Gudynas. 2000. Ecología del Paisaje en Uruguay. DINAMA y Junta de Andalucía. 173 pp.

Galante, E. \& A. Marcos-García. 1997. Detritívoros, coprófagos y necrófagos. Boletín de la Sociedad Entomológica Aragonesa, 20: 57-64.

Gaston, K. J. \& P. H. Williams. 1993. Mapping the world's biodiversity: the higher taxon approach. Biodiversity Letters, 1: 2-8.

Goñi, B., M. E. Martínez \& P. Daguer. 1997. Studies of two Drosophila (Diptera, Drosophilidae) communities from urban Montevideo, Uruguay. Revista Brasileira de Entomología, 41: 89-93.

Goñi, B., M. E. Martínez, V. L. S. Valente \& C. R. Vilela. 1998. Preliminary data of the genus Drosophila (Diptera, Drosophilidae) from Uruguay. Revista Brasileira de Entomología, 42: 131-140.

Greenberg, B. 1971. Flies and Disease, Volume 1. Princeton University Press, Princeton. 856 pp.

Greenberg, B. 1973. Flies and Disease, Volume 2. Princeton University Press, Princeton. 447 pp.

Guimarães, J. H. G., N. Papavero \& A. P. Prado. 1983. As miiases na região Neotropical: identificação, biología, bibliografía. Revista Brasileira de Zoologia, 1: 239-416.

Hammer, Ø., D. A. T. Harper \& P. D. Ryan. 2001. PAST: Paleontological statistics software package for education and data Analysis. Palaeontologia Electronica, 4: 9 pp. http://palaeo-electronica. org/2001_1/past/issue1_01.htm

Hanski, I. 1987. Nutritional ecology of dung and carrion-feeding insects, pp. 837-884. In: F. Slansky \& J. G. Rodriguez (Eds.) Nutritional ecology of insects, mites, spiders, and related invertebrates. Wiley, New York. 
Hernández-Ortiz, V. \& J. F. Dzul-Cauich. 2008. Moscas (Insecta: Diptera), pp: 95-105. In: R. H. Manson, V. Hernández-Ortiz, S. Gallina \& K. Mehltrether (Eds.). Agroecosistemas cafetaleros de Veracruz: biodiversidad, manejo y conservación. Instituto de Ecología, A.C. (INECOL) e Instituto Nacional de Ecología (INE-SEMARNAT), México.

Kearns, C. A. 2001. North American dipteran pollinators: Assessing their value and conservation status. Conservation Ecology, 5: 5.

Kuusela, S. \& I. Hanski. 1982. The structure of carrion fly communities: the size and type of carrion. Holartic Ecology, 5: 337-348.

Magurran, A. E. 1988. Diversidad ecológica y su medición. Ediciones Vedrá, Barcelona. 200 pp.

Mariluis, J. C., J. A. Schnack, P. R. Mulieri \& J. P. Torreta. 2007. The Sarcophagidae (Diptera) of the coastline of Buenos Aires City, Argentina. Journal of the Kansas Entomological Society, 80: 243-251.

Martínez, M. 2002. Los mosquitos anofelinos vectores del paludismo o malaria, pp. 91-94. In: Aber, A. (Ed.). Insectos y medio ambiente. Ministerio de Vivienda, Ordenamiento Territorial y Medio Ambiente, Montevideo.

Martínez-Sánchez, A., S. Rojo \& M. A. Marcos-García. 2000a. Annual and spatial activity of dung flies and carrion in a Mediterranean holm-oak pasture ecosystem. Medical and Veterinary Entomology, 14: 56-63.

Martínez-Sánchez, A., S. Rojo \& M. A. Marcos-García. 2000b. Sarcofágidos necrófagos y coprófagos asociados a un agroecosistema de dehesa (Diptera, Sarcophagidae). Boletín de la Asociación Española de Entomología, 24: 171-185.

McAlpine, D. K. 2007. Review of the Borboroidini or wombat flies (Diptera: Heteromyzidae), with reconsideration of the status of families Heleomyzidae and Sphaeroceridae, and descriptions of femoral gland-baskets. Records of the Australian Museum, 59: 143-219.

McAlpine, J. F., B. V. Peterson, G. E. Shewell, H. J. Teskey, J. R. Vockeroth \& D. M. Wood. 1981. Manual of Neartic Diptera. Vol. 1. Biosystematics Research Institute. Canada. 146 pp.

Morelli, E., P. González \& A. Baz. 2002. Coprophagous beteles in uruguayan prairies: abundance, diversity and seasonal ocurrence. Studies of Neotropical Fauna and Environment, 37: 53-57.

Morón, M. A. \& R. Terrón. 1984. Distribución altitudinal y estacional de los insectos necrófilos en la Sierra Norte de Hidalgo, México. Acta Zoológica Mexicana (n.s.), 3: 1-47.

Moura, M. O., C. J. Carvalho \& E. L. Monteiro-Filho. 1997. A preliminary analysis of insects of medicol-legal importance in Curitiba, State of Paraná. Memórias do Instituto Oswaldo Cruz, 92: 269-274.

Mulieri, P. R., J. P. Torreta, J. A. Schnack \& J. C. Mariluis. 2006. Calliphoridae (Diptera) of the coastline of Buenos Aires, Argentina: species composition, numerical trends and bait's preferences. Entomological News, 117: 139-146.

Pollet, M. 2009. Diptera as ecological indicators of habitat and habitat change, pp. 302-322. In: T. Pape, D. Bickel \& R. Meier (Eds.). Diptera diversity: status, challenges and tools. Brill, Leiden and Boston. 459 pp.

Porcile Maderi, J. F. 2005. El desafío del manejo sustentable del bosque nativo. Forestal, 15: 1-6.

Prance, G. T. 1994. A comparison of the efficacy of higher taxa and species numbers in the assessment of biodiversity in the neotropics. Philosophical Transactions of the Royal Society of London, Series B, 345: 89-99.

Putman, R. J. 1983. Carrion and dung: the decomposition of animal wastes. Edward Arnold, London. $62 \mathrm{pp}$.

Ratcliffe, B. C. 1996. The carrion beetles (Coleoptera: Silphidae) of Nebraska. University of Nebraska State Museum Bulletin 13: 100 pp. 
Rintoul, D. A., L. M. Krueger, C. Woodard \& J. E. Throne. 2005 Carrion Beetles (Coleoptera: Silphidae) of the Konza Prairie Biological Station. Journal of the Kansas Entomological Society, 78: 124-133.

Rossi, G. C. \& M. Martínez. 2003. Mosquitos (Diptera: Culicidae) del Uruguay. Entomología y Vectores, 10: $469 \cdot 478$.

Salvatella, R. 1997. Aedes aegypti (Diptera, Culicidae): notificación de su presencia en Uruguay. Revista Médica del Uruguay, 13: 118-121.

Skidmore, P. 1991. Insects of the Brithish cow-community. Field Studies Council. Occasional Publication $\mathrm{N}^{\circ} 21$, Shrewsbury, $166 \mathrm{pp}$.

Spinelli, G. R. \& M. Martínez. 1991. The genus Culicoides in Uruguay. (Diptera: Ceratopogonidae). Insecta Mundi, 5: 175-179.

Vogt, W. G., S. Runco \& N. T. Starick. 1985. A wind-oriented fly trap for quantitative sampling of adult Musca vetustissima Walker. Journal of the Australian Entomology Society, 24: 223-227.

Williams, P. H. \& K. J. Gaston. 1994. Measuring more of biodiversity: can higher taxon richness predict wholesale species richness? Biological Conservation, 67: 211-217. 